\title{
Modélisation numérique de l'interaction entaille-inclusion dans une plaque sollicitée
}

\author{
Djamel Ouinas ${ }^{1, a}$, Boualem Serier $^{2}$ et Bachir Bel abbes Bouiadjra ${ }^{2}$ \\ 1 Département de Génie-Mécanique, Faculté des sciences de l'ingénieur, Université de Mostaganem, 27000, Algérie \\ 2 Département de Génie-Mécanique, Faculté des sciences de l'ingénieur, Université de Sidi Bel Abbes, 22000, Algérie
}

Reçu le 4 juillet 2002, accepté le 3 décembre 2004

\begin{abstract}
Résumé - La compréhension du phénomène de la rupture des matériaux nécessite des études approfondies des phénomènes d'amorçage et de propagation des fissures qui, en général, prennent naissance dans les zones de fortes concentrations de contraintes dues aux effets géométriques ou métallurgiques. La connaissance de la distribution des contraintes au voisinage des différentes zones a une importance pour l'analyse de la variation du facteur de concentration de contraintes en fonction de leurs géométries. Ceci permet aussi d'expliquer le phénomène d'amorçage d'une rupture et de quantifier le facteur d'intensité de contraintes (FIC) en fonction de la géométrie de l'entaille et de la longueur de la fissure. Dans cette étude, on a essayé de modéliser par l'application de la méthode des éléments finis (MEF) l'effet de la présence d'une entaille et d'une inclusion sur le comportement d'une structure.
\end{abstract}

Mots clés : Rupture / entaille et fissure / propagation / inclusion / facteur d'intensité de contraintes (FIC) / interface matrice-inclusion

\begin{abstract}
The understanding of the fracture phenomena of materials requires through studies of initiation and propagation of cracks. Their initiations are, in general, localised in the zones of higth stress concentrations due to the geometric or metallurgic effects. The knowledge of the stress distribution to neighborhood of different zones has an importance role for analysis of the variation of stress concentration factor according to their geometries. It also permits to explain the phenomenon of crack initiation and to quantify the stress intensity factor (SIF) according to geometry of the notch and the crack length. In this study, one tried of modelling, by application of finite element method (FEM), the effect of the presence of a notch and an inclusion on the behavior of a structure.
\end{abstract}

Key words: Fracture / notch and crack / propagation / inclusion / stress intensity factor (SIF) / matrixinclusion interface

\section{Introduction}

De nombreux matériaux présentent des hétérogénéités microstructurales importantes telles que les inclusions, les cavités et les microfissures, etc. Le comportement élastique des matériaux est très affecté par la présence de défauts qui peuvent entraîner l'affaiblissement de la structure et provoquer sa destruction. Dans les zones à fortes concentrations de contraintes dues aux effets géométriques ou métallurgiques, des microfissures apparaissent ; ces microfissures se développent et coalescent les unes avec les autres jusqu'à ce qu'elles forment une fissure de taille macroscopique qui se propage jusqu'à la ruine de la structure.

a Auteur correspondant : rahouin@caramail.com
L'amorçage de la rupture ductile des matériaux s'effectue par décohésion de la matrice autour des inclusions, ou par fragmentation de celles-ci, avec formation de microcavités; ces cavités croissent et changent de forme sous l'effet de sollicitations mécaniques. Les inclusions sont généralement l'origine des concentrations de contraintes dans les matériaux et peuvent être une source d'amorçage et de propagation de fissures. De nombreux auteurs [1-6] se sont penchés sur l'étude de la présence d'inclusions; de même que plusieurs études [7-14] ont porté sur l'influence de l'effet d'entaille sur le comportement des matériaux.

\section{Modélisation par éléments finis}

Dans cette modélisation, on considère une plaque mince de longueur $H$ et de largeur $w$, elle est munie 
Tableau 1. Caractéristiques mécaniques de l'alliage d'aluminium de type 2024.

\begin{tabular}{cccc}
\hline$E[\mathrm{Mpa}]$ & $\sigma_{\mathrm{e}}[\mathrm{Mpa}]$ & $\nu$ & $K_{\mathrm{IC}}[\mathrm{Mpa} \sqrt{m}]$ \\
\hline 72395 & 425 & 0,33 & 38,5 \\
\hline
\end{tabular}

d'une entaille semi-circulaire latérale. Les rapports entre rayon de l'entaille $R$, la longueur $H$ et la largeur de la plaque $w$ sont respectivement $H / w=0,5$ et $R / w=0,1$. Les caractéristiques mécaniques du matériau étudié sont reportées dans le tableau 1.

La concentration de contraintes, permet d'augmenter la contrainte au fond de l'entaille pour atteindre la contrainte nécessaire en vue d'amorcer une fissure suivie d'une rupture. L'entaille est caractérisée par un facteur de concentration de contraintes $K_{\mathrm{t}}$ défini par Peterson $[15,16]$ de deux façons différentes :

La première mentionne le rapport de la contrainte maximale $\sigma_{\max }$ en fond d'entaille sur la contrainte nominale $\sigma_{\mathrm{N}}$ dans la section entaillée [17] :

$$
K_{\mathrm{t}}=\frac{\sigma_{\max }}{\sigma_{\mathrm{N}}}
$$

Dans la seconde version, le facteur $K_{\mathrm{t}}$ est défini par le rapport entre la contrainte maximale sur la contrainte appliquée $\sigma$ :

$$
K_{\mathrm{t}}=\frac{\sigma_{\max }}{\sigma}
$$

L'expression de la contrainte longitudinale $\sigma_{Y}$ en amont du point d'entaille d'une plaque infinie [18] où $K_{\mathrm{t}}=3$ est comme suit :

$$
\sigma_{Y}=\sigma_{\mathrm{N}}\left[1+\frac{1}{2}\left(1+\frac{x}{\rho}\right)^{-2}+\frac{3}{2}\left(1+\frac{x}{\rho}\right)^{-4}\right]
$$

Pour les corps sans entailles soumis à une tension uniforme [13], le facteur $K$ pour le point le plus profond de la surface de petite fissure est donné par la relation suivante :

$$
K=Q \sigma \sqrt{\pi a}
$$

Dans le cas d'une fissure émanant du point d'entaille, l'expression (4) doit être modifiée en remplaçant $\sigma$ par la distribution de la contrainte locale donnée par la relation (3) à une distance $x=a$.

$$
K=Q f \frac{K_{\mathrm{t}} \sigma_{\mathrm{N}}}{2}\left[\left(1+2 \frac{a}{\rho}\right)^{-1 / 2}+\left(1+2 \frac{a}{\rho}\right)^{-3 / 2}\right]
$$

avec $f$ désignant le facteur de correction qui dépend de $K_{\mathrm{t}}$ et $a$.

Le facteur de correction $f$ varie respectivement avec l'augmentation du rapport géométrique $x / \rho$ et le facteur de concentration de contraintes, ce facteur peut être déterminé par les expressions suivantes :

$$
\begin{cases}f=1 & \text { pour } \frac{a}{\rho}<0,2 \\ f=1+\frac{\operatorname{tg}\left(\pi / 2 K_{\mathrm{t}}\right)}{2,8}\left(\frac{a}{\rho}-0,2\right) & \text { pour } \frac{a}{\rho} \geq 0,2\end{cases}
$$

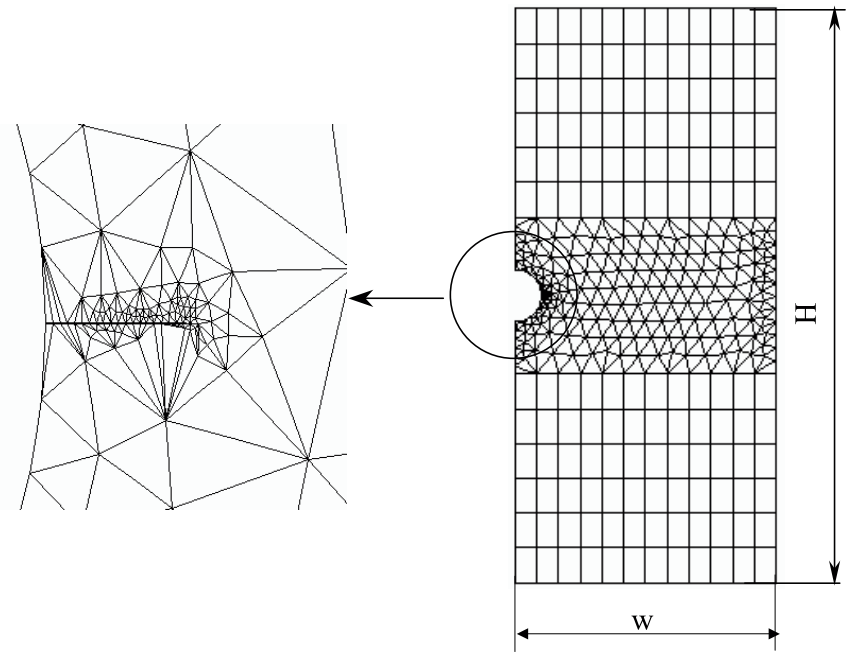

Fig. 1. Maillage utilisé dans la modélisation.

Dans les travaux de Kujawski [11], le facteur $K_{\text {I }}$ est calculé à partir de $K_{\mathrm{t}}$ et du rayon de l'entaille $\rho$; pour une éprouvette à entaille semi-circulaire latérale, le facteur $K_{\mathrm{I}}$ prend la forme suivante :

$$
\begin{aligned}
& \frac{K}{1,122 \sigma \sqrt{\pi a}}= \frac{f}{1-1 / K_{\mathrm{t}}}\left[\left(1+2 \frac{a}{\rho}\right)^{-\frac{1}{2}}\right. \\
&\left.+\left(1+2 \frac{a}{\rho}\right)^{-\frac{3}{2}}\right] \sqrt{\frac{a}{\rho}} \\
& \frac{K}{1,122 \sigma \sqrt{\pi a}}=\sqrt{1+\frac{a}{\rho}\left(\frac{2}{K_{\mathrm{t}}-1}\right)^{2}}
\end{aligned}
$$

Avec « $a »$ désignant la profondeur de l'encoche.

\section{Facteur d'intensité de contraintes}

On a confronté les résultats du calcul du FIC obtenus en utilisant le code de calcul FRANC-2D/L [19] pour des fissures émanant de l'entaille semi-circulaire latérale avec ceux obtenus par simulation du modèle de Kujawski [11] établis pour les fissures courtes émanant des fonds d'entaille. La plaque considérée est chargée en traction uniaxiale dans la direction verticale $Y$ sous la contrainte appliquée d'amplitude $\sigma=10 \mathrm{MPa}$ avec une fissure de longueur $a=1,2 \mathrm{~mm}$ amorcée au fond de l'entaille (voir Fig. 1).

Le maillage au niveau de l'entaille et autour de la fissure est bien affiné en faisant varier la longueur de la fissure. Les résultats obtenus expriment la variation du FIC normalisé $K_{\mathrm{I}} / \sigma \sqrt{\pi a_{\mathrm{P}}}\left(a_{\mathrm{p}}=a+R\right)$ en fonction des différentes longueurs normalisées de la fissure $a / R$ et sont reportés dans la figure 2 .

La comparaison de ces résultats avec ceux du modèle de Kujawski [11] montre une très bonne coïncidence pour les fissures courtes puisque, dans tous les cas, l'écart ne dépasse pas les « $6 \%$ ». À noter que la modélisation des fissures courtes est très délicate à mettre en ouvre. 


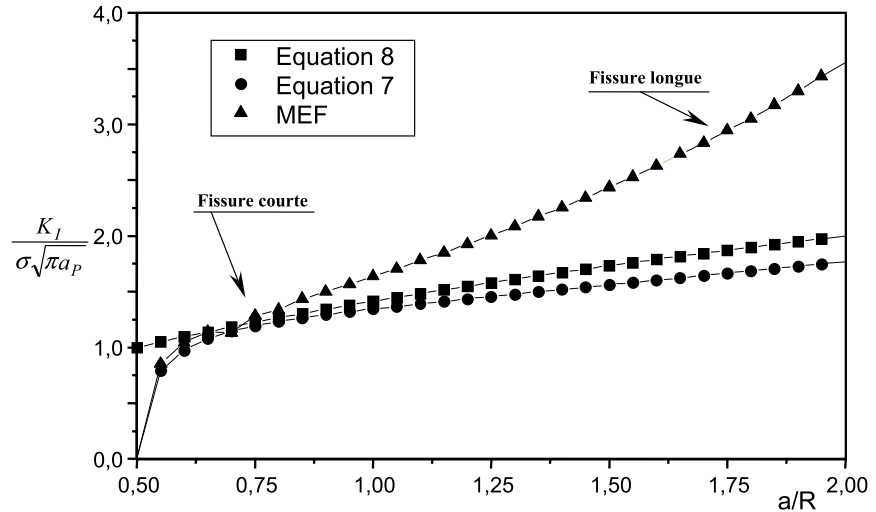

Fig. 2. Comparaison des résultats du FIC obtenus par le modèle (Éqs. (7) et (8)) et la MEF.
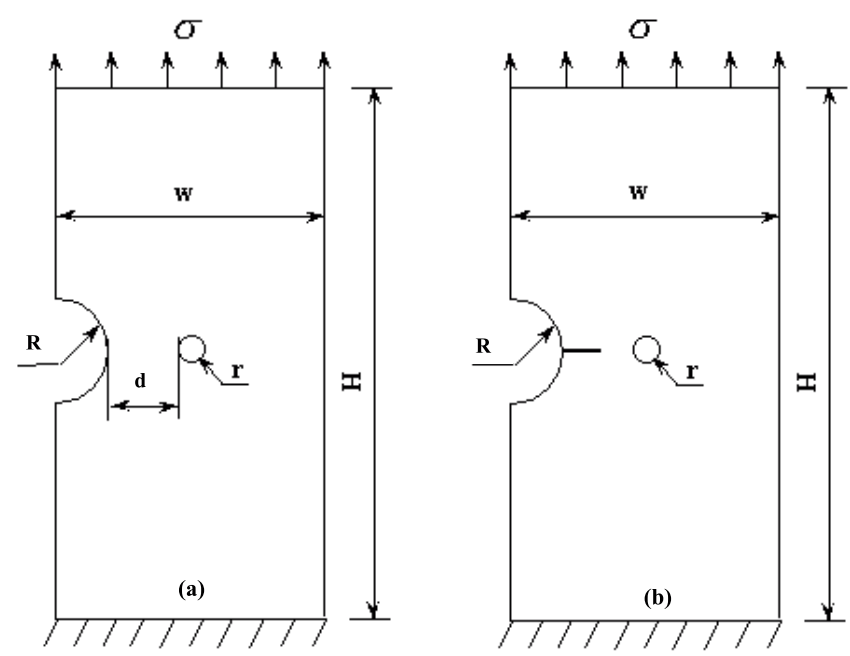

Fig. 3. Représentation d'une plaque sollicitée en traction en présence d'une inclusion sur le ligament de la plaque.

\section{Interaction entaille-inclusion}

On considère le même modèle géométrique avec le même chargement dont les propriétés du matériau constituant la plaque sont : le module de Young $E_{1}$ et le coefficient de Poisson $\nu_{1}$ (Fig. 3) contenant une inclusion de configuration sphérique de rayon $« r »$ située à une distance $« d »$ de l'entaille. Les propriétés du matériau de l'inclusion sont : le module de Young $E_{2}$ et le coefficient de Poisson $\nu_{2}$ et $r / R=0,1$ et $\nu_{1}=\nu_{2}=0,25$. La figure $3 \mathrm{a}$ montre le modèle géométrique de la plaque sans fissure. Dans une seconde approche, on prévoit l'existence d'une fissure émanant du fond de l'entaille semi-circulaire comme le montre la figure $3 \mathrm{~b}$.

\subsection{Inclusion située au fond de l'entaille}

On considère que l'inclusion est adjacente à l'entaille dont l'épaisseur de l'interface tend vers zéro. La variation des contraintes normales au voisinage de l'entaille et de l'inclusion en fonction du rapport des modules de Young : inclusion-matrice est représentée sur la figure 4 .
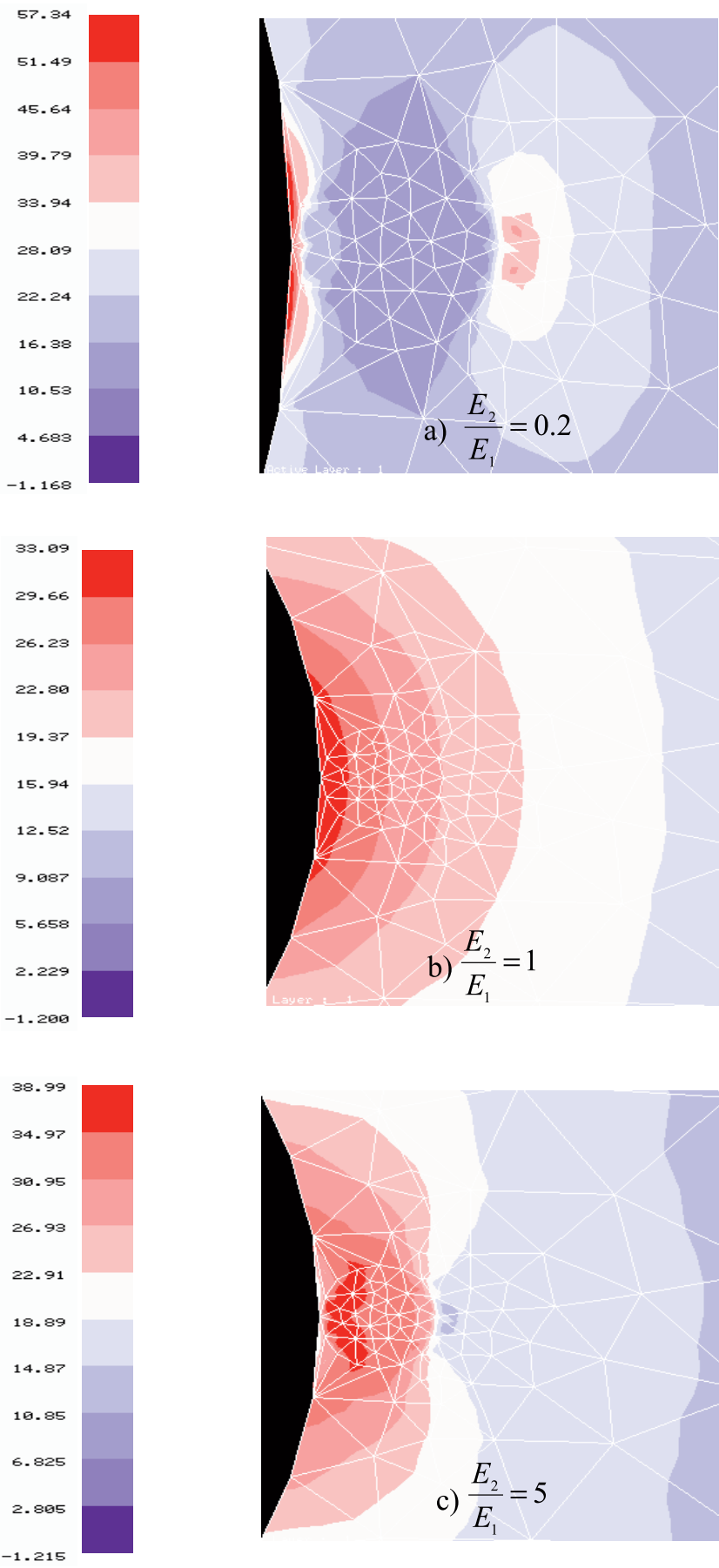

Fig. 4. Variation du champ de contraintes normales au niveau $\mathrm{du}$ fond d'entaille et de l'inclusion pour différents rapports $E_{2} / E_{1}$.

On constate que lorsque l'inclusion est plus dure que la matrice $\left(E_{2} / E_{1}=5\right)$, les contraintes maximales sont obtenues au niveau de l'entaille et de l'inclusion; ceci montre que la présence de ce type d'inclusion fragilise la structure et peut conduire à l'augmentation du risque d'amorçage des fissures. Ce dernier peut donc avoir lieu soit au fond de l'entaille soit au niveau de l'inclusion (Fig. 4c). 


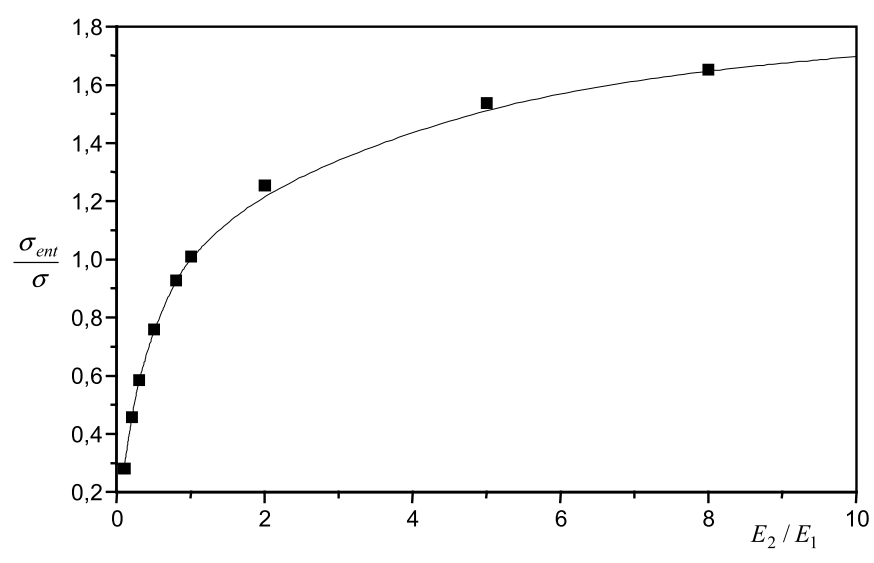

Fig. 5. Variation du champ de contraintes normales au niveau du fond d'entaille pour différents rapports $E_{2} / E_{1}$.

L'effet de l'inclusion est sensible lorsqu'elle est du même module de Young que la matrice. Les contraintes les plus élevées sont réparties au niveau de l'entaille (Fig. 4b).

Lorsque l'inclusion présente des propriétés de ductilité plus importantes que celles de la matrice $\left(E_{2} / E_{1}=0,2\right)$ les contraintes maximales sont réparties de part et d'autre $\mathrm{du}$ fond de l'entaille et les contraintes minimales sont concentrées à l'intérieur de l'inclusion. La contrainte au fond de l'entaille est un peu plus faible. Donc une inclusion moins dure que la matrice conduit à une structure moins fragilisée et à de faibles risques d'amorçage de fissure.

Pour une meilleure étude de l'effet des propriétés mécaniques de l'inclusion, on représente sur la figure 5 l'évolution de la contrainte au fond de l'entaille en fonction du rapport des modules de Young matriceinclusion $E_{2} / E_{1}$.

On remarque que la contrainte au fond de l'entaille croît avec le rapport $E_{2} / E_{1}$; ceci confirme les résultats présentés sur la figure 4 . Cet accroissement est très marqué pour des rapports $E_{2} / E_{1}$ inférieurs à deux (2) tandis que pour des valeurs supérieures à ce nombre, l'évolution des contraintes au niveau de l'entaille devient peu sensible; ceci s'explique par le fait que lorsque les propriétés de résistance de l'inclusion sont beaucoup plus élevées que celles de la matrice, les concentrations de contraintes sont au niveau de l'inclusion.

Les résultats représentés sur la figure 6 sont obtenus pour trois rapports $E_{2} / E_{1}$. On remarque que pour un rapport $E_{2} / E_{1}=0,2$ la contrainte maximale est au niveau de l'interface matrice-inclusion (point i, Fig. 6) et beaucoup moins importante à l'interface inclusion-entaille (point h, Fig. 6).

Une inclusion moins dure que la matrice permet l'obtention d'une concentration de contraintes de degré moindre à cause de l'effet d'absorption de l'énergie appliquée sous forme de déformation élastique ou plastique; un effet contraire est observé pour un rapport égal à cinq $\left(E_{2} / E_{1}=5\right)$. Dans ce cas les contraintes minimales sont au niveau de l'interface matrice-inclusion. Lorsque la matrice et l'inclusion ont les mêmes caractéristiques

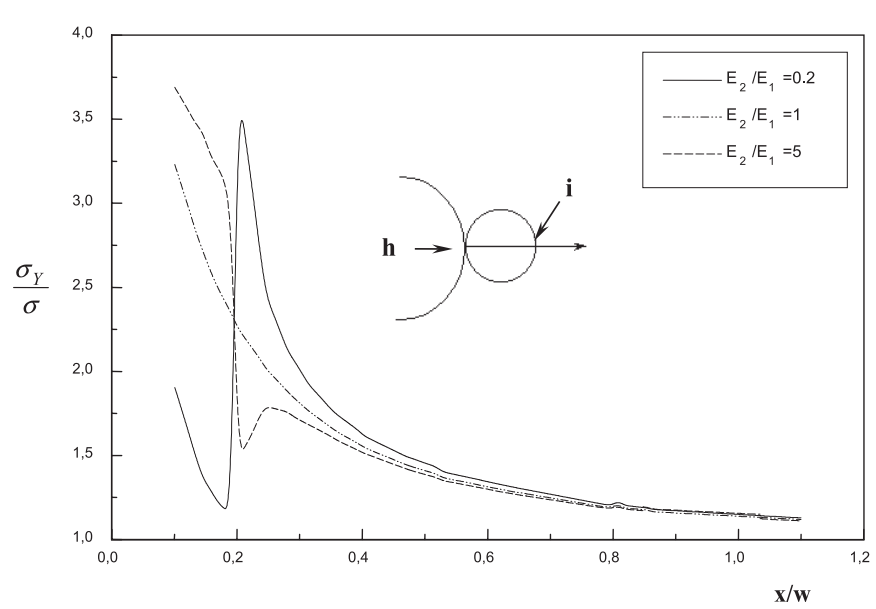

Fig. 6. Variation du champ de contraintes normales sur le ligament de la plaque en présence de l'inclusion en fond de l'entaille.

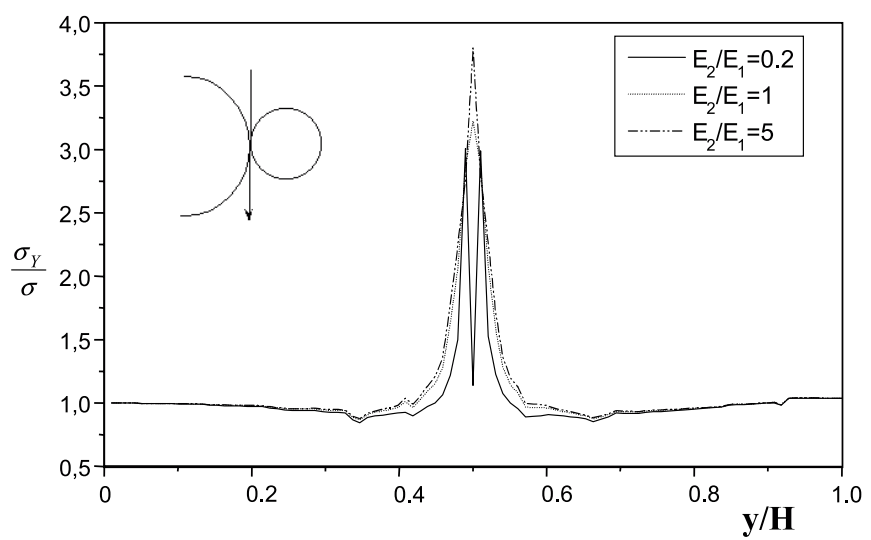

Fig. 7. Variation du champ de contraintes normales sur la verticale en présence de l'inclusion en fond de l'entaille.

mécaniques $\left(E_{2} / E_{1}=1\right)$ un seul effet est prépondérant c'est celui de l'entaille; la matrice est donc continue.

La figure 7 montre que pour $E_{2} / E_{1}=5$ et $E_{2} / E_{1}=1$, la contrainte est maximale au fond de l'entaille et s'accentue davantage pour $E_{2} / E_{1}=5$.

Si la matrice est plus dure que l'inclusion $\left(E_{2} / E_{1}=\right.$ 0,2 ) la contrainte augmente en s'approchant du fond de l'entaille puis diminue au point caractérisant ce fond, ceci est dû aux propriétés mécaniques de l'inclusion.

\subsection{Inclusion distante du fond de l'entaille}

Afin de mettre en évidence l'effet de la position de l'inclusion par rapport à l'entaille, on considère une inclusion distante de l'entaille permettant d'avoir des rapport $d / R=2$.

On représente, sur la figure 8, la variation des contraintes normales sur le ligament en présence d'une inclusion et on montre que quelque soit la valeur du rapport $E_{2} / E_{1}$, les contraintes maximales sont au niveau de l'entaille; ces contraintes diminuent au fur et à mesure que l'on avance vers l'inclusion, puis augmentent ou 


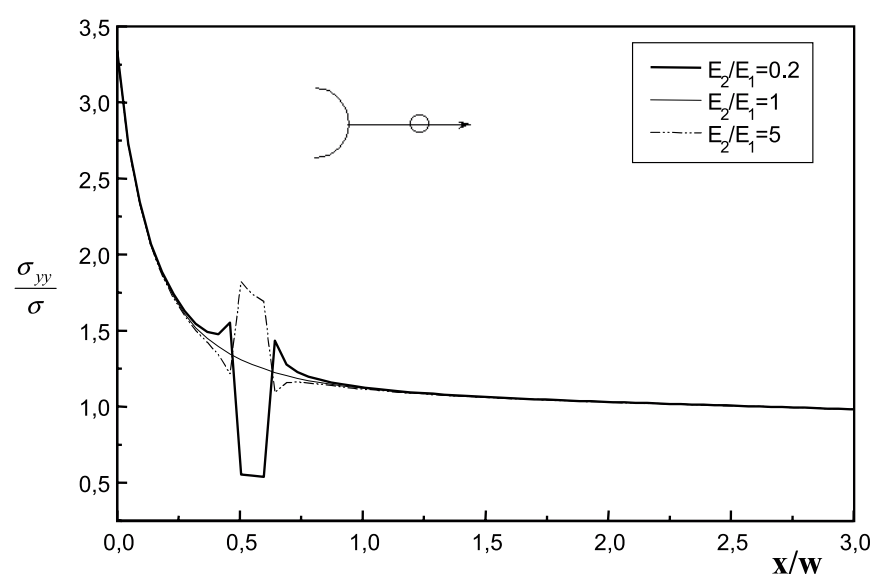

Fig. 8. Variation du champ de contraintes normales en présence de l'inclusion sur le ligament.

diminuent à l'interface inclusion-matrice en fonction des différentes caractéristiques mécaniques inclusion-matrice et de leurs résistances.

\section{Interaction de la fissure émanant de l'entaille-inclusion}

Pour mener à bien cette étude on considère que la plaque contient une fissure émanant de l'entaille et une inclusion. Deux cas ont été analysés : une inclusion située au fond de l'entaille et une inclusion située à une certaine distance de l'entaille.

\subsection{Inclusion située au fond de l'entaille}

Dans ce cas, la fissure se trouve dans l'inclusion et se propage vers la matrice; on détermine la variation du FIC normalisé $K_{\mathrm{I}} / K_{0}\left(K_{0}=\sigma \sqrt{\pi a}\right)$ en fonction des différents rapports des modules de Young inclusion-matrice.

Les résultats obtenues correspondent aux différentes longueurs de fissure respectivement $a=0,75,1,25$ et $2 \mathrm{~mm}$; ces résultats sont représentés sur la figure 9 .

On remarque que le FIC normalisé croît avec le rapport des modules de Young $E_{2} / E_{1}$ et que pour une même valeur de $E_{2} / E_{1}$ une augmentation de la longueur de la fissure entraîne un accroissement du FIC, cela signifie qu'une augmentation de ce rapport conduit à une énergie plus importante en tête de fissure.

Sur la figure 10, on présente la variation du FIC normalisé en fonction du rapport $d / a_{\mathrm{P}}$ de la distance séparant l'inclusion de la pointe de la fissure pour trois valeurs du rapport $E_{2} / E_{1}$. La longueur de la fissure varie en fonction de sa propagation jusqu'à l'interface inclusionmatrice.

Lorsque la fissure se propage dans l'inclusion, la distance $« d »$ diminue. Il est à noter, cependant, que le FIC normalisé $K_{\mathrm{I}} / K_{0}$ augmente très sensiblement avec le rapport $d / a_{\mathrm{P}}$ pour un rapport $E_{2} / E_{1}=5$; sa valeur maximale est atteinte à l'interface inclusion-matrice. La

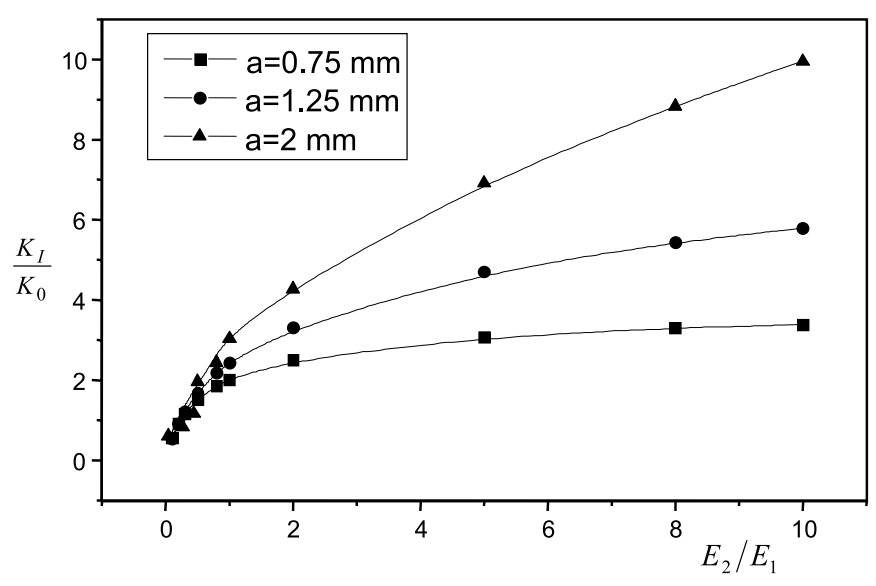

Fig. 9. Variation du FIC en fonction des caractéristiques des matériaux inclusion-matrice.

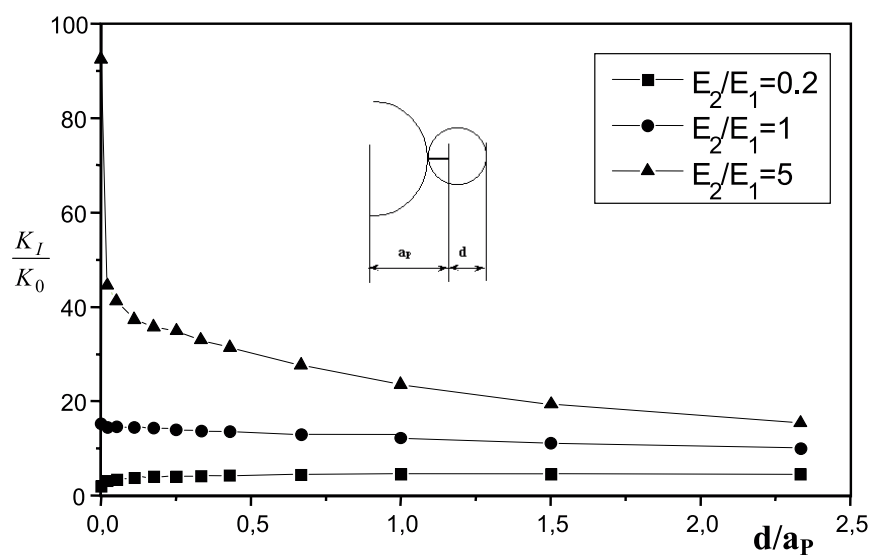

Fig. 10. Variation du FIC en fonction de l'avance de la fissure dans l'inclusion.

fissure se propage vers le matériau le moins dur (matrice) et son énergie s'affaiblit au fur et à mesure que la fissure s'éloigne de l'interface.

Pour un rapport $E_{2} / E_{1}=0,2$, le FIC normalisé diminue avec l'augmentation du rapport $d / a_{\mathrm{P}}$; dans ce cas l'énergie en tête de fissure diminue lorsque la fissure approche de l'interface. Si la matrice et l'inclusion présentent les mêmes caractéristiques mécaniques $\left(E_{2} / E_{1}=1\right)$, on peut considérer qu'il s'agit du même matériau et c'est l'effet de la fissure qui apparaît et se propage dans la matrice avec une énergie constante.

\subsection{Inclusion distante du fond de l'entaille}

Dans ce cas on suppose que la fissure est située dans l'entaille de la matrice et se propage vers l'inclusion distante de $« d »$. Les niveaux de contraintes ainsi obtenus pour les trois comportements $E_{2} / E_{1}$ et pour un rapport $d / a \approx 0$ sont représentés sur la figure 11. Cette figure montre clairement, que pour un rapport $E_{2} / E_{1}=5$, les contraintes maximales sont localisées en avant de la tête de la fissure; et l'effet contraire est observé lorsque le rapport devient égal à $0,2\left(E_{2} / E_{1}=0,2\right)$. Il faut noter, 

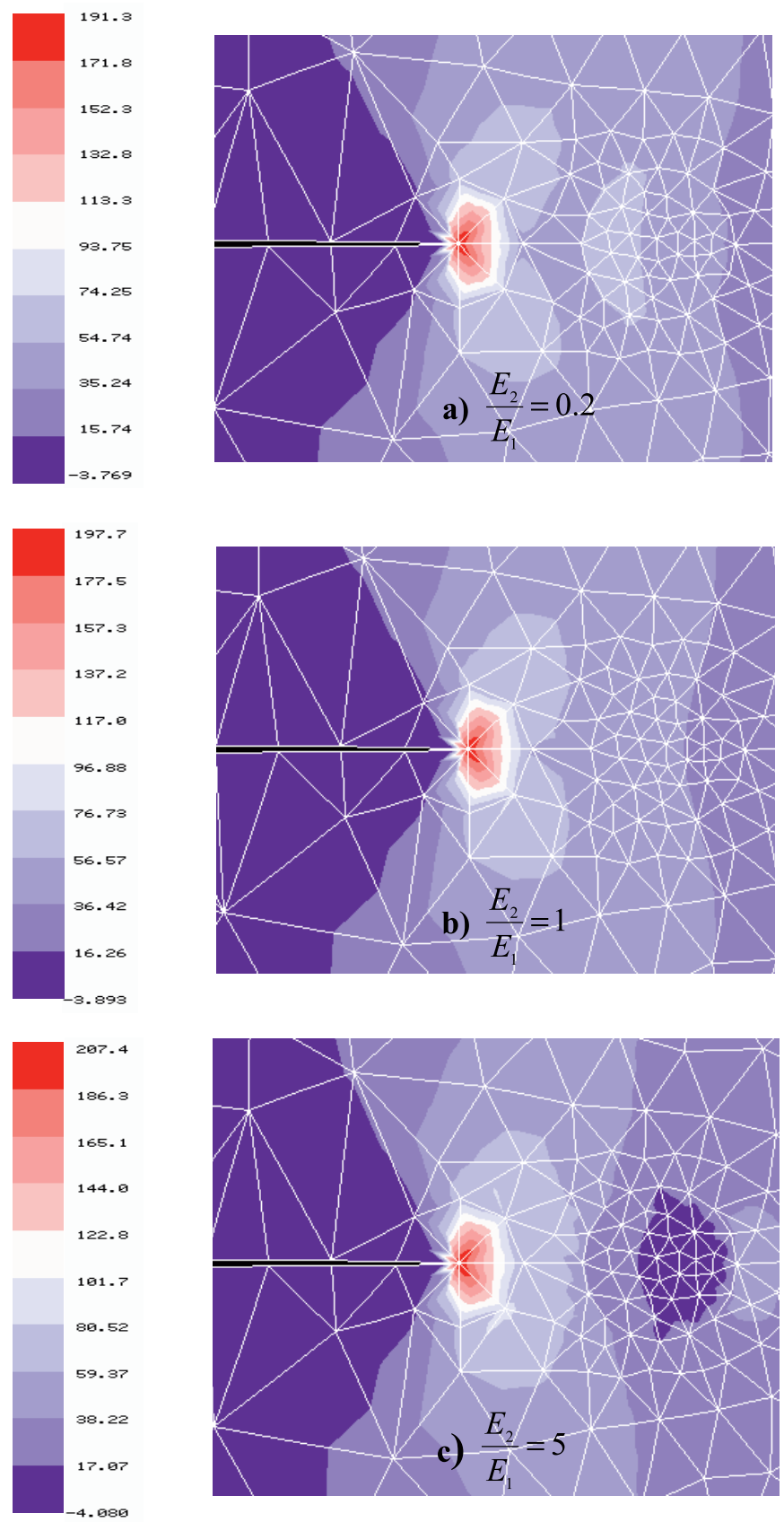

Fig. 11. Variation du champ de contraintes normales au niveau de la pointe de la fissure qui se propage vers l'inclusion pour différents rapports $E_{2} / E_{1}$.

cependant, que ce phénomène ne fait son apparition que lorsque la fissure est très proche de l'inclusion.

On peut dire que cet effet est globalement dû à l'interaction fissure-inclusion; autrement dit, une inclusion moins dure que la matrice permet le ralentissement de la propagation de la fissure, voire même son arrêt. Dans le cas où l'inclusion est plus dure que la matrice $\left(E_{2} / E_{1}=\right.$ 5 ), elle sera source d'une propagation brutale de fissure dite rupture fragile.

Dans le but de compléter cette étude, on s'est penché sur le comportement du FIC normalisé $K_{\mathrm{I}} / K_{0}$ en fonction

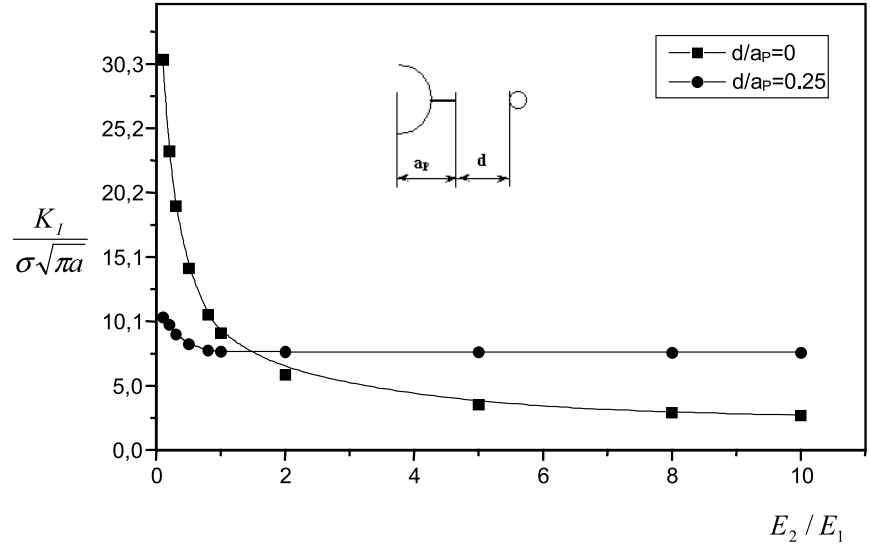

Fig. 12. Variation du FIC en fonction des caractéristiques des matériaux inclusion-matrice.

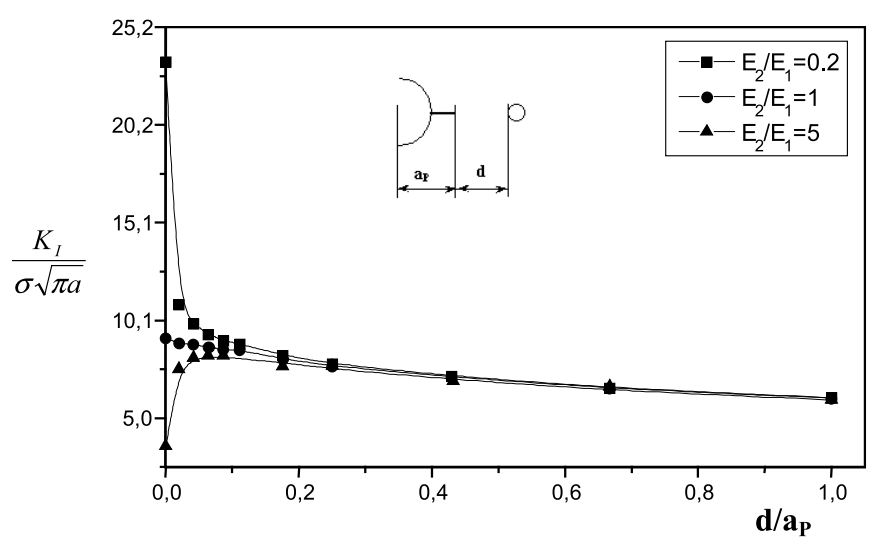

Fig. 13. Variation du FIC en fonction de l'avance de la fissure vers l'inclusion.

des rapports des modules de Young inclusion-matrice $\left(E_{2} / E_{1}\right)$ pour deux rapports $d / a_{\mathrm{P}}=0$ et $d / a_{\mathrm{P}}=0,25$; ce comportement est illustré sur la figure 12 . On constate que pour $d / a_{\mathrm{P}}=0$ le FIC normalisé croît brutalement lorsque le rapport $E_{2} / E_{1}$ diminue; cette augmentation du FIC est beaucoup plus marquée pour des rapports inférieurs à $2\left(E_{2} / E_{1}<2\right)$. L'évolution du FIC avec la diminution du rapport $\left(E_{2} / E_{1}\right)$ devient peu sensible lorsque l'inclusion est située à certaine distance de la fissure et l'effet de l'interaction inclusion-fissure disparaît.

L'influence des propriétés de résistance de l'inclusion, de la matrice et $\mathrm{du}$ rapport $d / a_{\mathrm{P}}$ sur le comportement du FIC normalisé a été également étudiée. Les résultats sont représentés sur la figure 13 . On remarque que le comportement du FIC normalisé est contraire à celui qui est représenté sur la figure 10.

Le FIC normalisé augmente avec le rapport $d / a_{\mathrm{P}}$ pour des valeurs $E_{2} / E_{1}=1$ et $E_{2} / E_{1}=0,2$ et diminue lorsque $E_{2} / E_{1}=5$. Cette variation est plus importante pour des valeurs $d / a_{\mathrm{P}}<0,25$. Lorsque le rapport $d / a$ tend vers zéro, le FIC normalisé tend vers une valeur nulle, ce qui montre clairement qu'une inclusion plus dure peut freiner la propagation de la fissure. 


\section{Conclusion}

Cette étude a été menée dans le but d'étudier le comportement d'une structure entaillée sous l'effet de la présence d'une inclusion près de l'entaille. L'analyse des résultats donnés par la méthode des éléments finis a permis de tirer les conclusions suivantes :

- La présence d'une inclusion près de l'entaille augmente la fragilité de la structure ainsi que le risque d'amorçage des fissures; les inclusions sont dans la plus part des cas des oxydes ou des sulfures, elles sont donc plus dures que les matrices.

- L'énergie créée en tête de fissure diminue lorsque la dureté de l'inclusion augmente si la fissure se trouve dans la matrice; et le phénomène inverse se produit si la fissure se trouve dans l'inclusion; son énergie augmente avec la dureté de l'inclusion.

- Lorsque la fissure se propage de la matrice vers l'inclusion, son énergie diminue à l'approche de l'interface, elle a tendance, soit à s'arrêter soit à changer de direction de propagation.

- Lorsque la fissure se propage de l'inclusion vers la matrice, son énergie augmente à l'approche de l'interface, elle a tendance à s'accélérer.

\section{Références}

[1] J.D. Eshelby, Proc. Roy. London Serie A241 (1957) 376-396

[2] D. Brooksbank, K.W.J. Andrew, Iron Steel Inst., June (1968) 595-599

[3] Y. Murakami, H. Usuki, Int. J. Fatigue 11 (1989) 299-307
[4] D.H. Chen, S. Nakamichi, Int. J. Fracture 82 (1996) 131-152

[5] A. Melander, Int. J. Fatigue 19 (1997) 13-24

[6] P. Lukas, M. Klesnil, Fatigue limit of notched bodies, Mater. Sci. Engng. 34 (1978) 61-66

[7] J.C. Newman Jr, An improved method of collocation for the stress analysis of cracked plates with various shaped boundaries, NASA TN D-6376, 1971

[8] J. Schive, Fatigue Engng. Mater. Struct. 5 (1982) 77-90

[9] G. Glinka, Engng. Fract. Mech. 22 (1983) 839-845

[10] G.C. Sih, Handbook of Stress Intensity Factors, Lehigh University, Bethehem, PA, 1973

[11] D. Kujawski, Estimations of Stress Intensity Factors For Small cracks at Notches, Fatigue Fract., Engng. Mater. Struct. 14 (1991) 953-965

[12] P. Lukas, Stress intensity factor for small notchemanating cracks, Engng. Fract. Mech. 26 (1987) 471-473

[13] Usami, short crack fatigue properties and component life estimation, Current research on Fatigue cracks, T. Tanaka, M. Jono, K. Komai (ed.), 1985

[14] J.C. Newman Jr, E.P. Phillips, R.A. Everett Jr, Fatigue Analysis Under Contant and Variable-Amplitude Loading Using Small Crack Theory, Mech. Mat. Branch, NASA Langley Research Center Hampton, Virginia USA 23681, NASA/TM-1999-209329

[15] R.E. Peterson, Notch Sensitivity, Metal Fatigue, G. Sines, J. Lwaisman (ed.), Mc Graw Hill, New York, 1959, pp. 293-306

[16] R.E. Peterson, Stress concentration factors, John Wiley \& Sons, New York, 1974

[17] T. Tamine, Amorçage de fissures par fatigue-contact, Thèse, Université de Metz, 1994

[18] Thimoshenko, J.N. Goodier, Theory of Elasticity, McGraw-Hill, New York, 1951

[19] FRANC-2D/L, User's Guide a two dimensional crack propagation simulator 1998

Retrouvez nos articles sur le site : www.edpsciences.org/meca 